\title{
Incentive compatibility and information superiority of the core of an economy with differential information*
}

\author{
Leonidas C. Koutsougeras and Nicholas C. Yannelis \\ Department of Economics, University of Illinois at Urbana-Champaign, Champaign, IL 61820, USA
}

Received: October 31, 1991; revised version March 12, 1992

Summary. We analyse the coarse, the fine, and the private core allocation of an exchange economy with differential information. The basic questions that we address are whether the above concepts are: (i) coalitionally incentive compatible, i.e., does truthful revelation of information in each coalition occur; and (ii) taking into account the information superiority or information advantage of an agent. Moreover, the above three concepts are examined in the presence of externalities and a comparison and interpretation of all of these core notions is provided.

\section{Introduction}

The idea of an exchange economy with differential information [i.e., an economy consisting of a finite set of traders each of whom is characterized by a state dependent (random) utility function, a random initial endowment, a private information set, and a prior], was introduced by Radner (1968) [we will sometimes call such an economy as a Radner-type economy]. The equilibrium notion that Radner (1968) adopted to analyze trade among agents in an economy with differential information was the Walrasian equilibrium. Since the Walrasian equilibrium notion is noncooperative it precludes cooperation among groups of agents. Thus, we adopt the core, a cooperative solution concept, in order to analyze the trade among agents in a Radner-type economy. We will argue that not only does the core provide more sensible outcomes than the Walrasian equilibrium, but it is also coalitionally incentive compatible (i.e., there is truthful revelation of information in each coalition) and it takes into account explicitly the information advantage or superiority of an agent.

Throughout the paper we will denote the private information set of agent $i$ (which is going to be a partition of a measure space) by $\mathscr{F}_{i}$. We will first examine versions of the coarse core of Wilson (1978), where the blocking net trades of a

\footnotetext{
* We wish to thank Stefan Krasa, Frank Page, Wayne Shafer, Anne Villamil, and Myrna Wooders for several useful comments, discussions, and suggestions. The comments of two referees were also helpful and we thank them for their careful reading. Obviously, we are responsible for any remaining errors.
} 
coalition $S$ are $\bigwedge_{i \in S} \mathscr{F}_{i}$-measurable ${ }^{1}$ and therefore the information is common knowledge to each member of the coalition. We next examine the fine core concept of Wilson (1978), where the blocking net trades of a coalition $S$ are $\underset{i \in S}{V} \mathscr{F}_{i-}$ measurable and hence the information is pooled by the members of the coalition. Finally, we examine the private core of Yannelis (1991), where the blocking net trade of each member of the coalition is $\mathscr{F}_{i}$-measurable and thus there is bargaining under differential information among the members in each coalition, contrary to the coarse and fine core.

We will show that the coarse core exists, it is coalitionally incentive compatible (i.e., there is truthful revelation of information in each coalition) and it takes into account the information superiority of an individual. However, since the coarse core always contains the private core and the latter exists and has the above properties, we will conclude that we learn nothing new from the coarse core that cannot be learned from the private core. In fact as we will show by means of an example, the coarse core is "too large," i.e., all the individually rational and Pareto optimal allocations constitute the coarse core.

Contrary to the coarse core, the basic problem with the fine core is that it is "too small" and in general it does not exist. Moreover, whenever it does exist we will show that it is not coalitionally incentive compatible and it does not take into account the information superiority of an individual. The analysis of these core concepts suggests that the private core may be the appropriate core notion in an exchange economy with differential information. This concept exists under standard continuity and concavity assumptions on the utility functions, it is coalitionally incentive compatible, and takes into account the information superiority of an agent. Moreover, we show that the private core can be used to model the idea of an intermediary [see Boyd-Prescott (1986) as well]. The intermediary is an agent with "better" information than all other agents who by using his/her superior information, executes the correct trades. The idea of an intermediary arises endogenously and naturally in our framework. Our results suggest that cooperative solution concepts may be quite useful for analyzing trade in economies with differential information and may be useful for tackling basic issues in the theory of financial markets.

The paper is organized as follows: Section 2 contains notation and the economic model. Several core notions are defined in Sect. 3 and some preliminary results are proved as well. Section 4 focuses mainly on the incentive compatibility of the private core. The interpretation of the different core concepts is given in Sect. 5 . Section 6 introduces different core notions in the presence of externalities, and an existence result is proved in Sect. 7 . Section 8 contains some concluding remarks. Finally, in Sect. 9 we compare the core with the value allocation of KrasaYannelis (1991).

\footnotetext{
${ }^{1}$ The symbol $\bigwedge_{i \in S} \mathscr{F}_{i}$ denotes the "meet," i.e., the maximal partition contained in all $\mathscr{F}_{i}$. The symbol $\vee \mathscr{F}_{i}$ denotes the "join," i.e., the minimal partition containing all $\mathscr{F}_{i}$. By an abuse of notation we $i \in S$ will denote throughout the paper, the $\sigma$-algebra generated by the partition $\mathscr{F}_{i}$ also by $\mathscr{F}_{i}$.
} 


\section{Notation and the economic model}

Before we outline our model we begin with some notation.

\subsection{Notation}

$\mathbb{R}^{n} \quad$ denotes the $n$-dimensional Euclidean space.

$\mathbb{R}_{+}^{n} \quad$ denotes the positive cone of $\mathbb{R}^{n}$.

$\mathbb{R}_{++}^{n}$ denotes the strictly positive cone of $\mathbb{R}^{n}$.

$2^{A}$ denotes the set of all subsets of the set $A$.

$\varnothing \quad$ denotes the empty set.

$\backslash$ denotes set-theoretic subtraction.

$|A|$ denotes the cardinality of the set $A$.

If $(\Omega, \mathscr{F}, \mu)$ is a measure space, then $\mathscr{F}_{i}$ will always denote a measurable partition of $\Omega$ (or a sub- $\sigma$-algebra) and $E_{i}(\omega)$ will denote the element of the partition $\mathscr{F}_{i}$ which contains $\omega \in \Omega$. If $X$ is a linear topological space, its dual is the space $X^{*}$ of all continuous linear functionals on $X$.

\subsection{The exchange economy with differential information}

Let $Y$ denote the commodity space. For simplicity one may identify $Y$ with the positive cone of $\mathbb{R}^{\prime}$. However, all the results in this paper remain true if $Y$ is the positive cone of any Banach lattice with an order continuous norm. ${ }^{2}$ Therefore, one can allow for infinitely many commodities. Denote by $(\Omega, \mathscr{F}, \mu)$ a probability measure space. An exchange economy with differential information $\mathscr{E}$ is given by

$\mathscr{E}=\left\{\left(X_{i}, u_{i}, e_{i}, \mathscr{F}_{i}, \mu\right): i=1,2, \ldots, n\right\}$ where

(1) $X_{i}: \Omega \rightarrow 2^{Y}$ is the consumption set of agent $i$,

(2) $u_{i}: Y \rightarrow \mathbb{B}$ is the utility function of agent $i$,

(3) $\mathscr{F}_{i}$ is a (measurable) partition of $\Omega$ denoting the private information ${ }^{3}$ of agent $i$,

(4) $e_{i}: \Omega \rightarrow Y$ is the initial endowment of agent $i$, where $e_{i}$ is $\mathscr{F}_{i}$-measurable, (Bochner) integrable and $e_{i}(\omega) \in X_{i}(\omega) \mu$-a.e.

(5) $\mu$ is a probability measure on $\Omega$ denoting the common prior of each agent.

The expected utility of agent $i$ is given by

$$
\int_{\omega \in \Omega} u_{i}\left(x_{i}(\omega)\right) d \mu(\omega)
$$

A possible interpretation of the above economy is the following: one may think that there are two periods where actual consumption takes place in the second period. In period one there is uncertainty over the states of nature and in this

\footnotetext{
${ }^{2}$ See Section 7 for rigorous definitions.

${ }^{3}$ As in Kobayashi (1980) we will assume that the members of a coalition release their private information sets honestly, i.e., private information sets are common knowledge.
} 
period agents make agreements which may be contingent on the realized state of nature in the second period. It is important to note that in this setting agents have differential information with respect to the realized state of nature and know their endowment realization (i.e., the initial endowment of each agent is $\mathscr{F}_{i}$-measurable). Note that a common prior assumption has been adopted in this framework. However, as in Yannelis (1991), one may allow for different priors, Bayesian updating, and random utility functions. All the results of this paper remain valid in this case, but we choose not to adopt the latter modeling for simplicity of exposition and easier calculation of our examples introduced later in the paper.

\section{Core notions and preliminary results}

In this section we define several different core notions for an exchange economy with differential information.

\subsection{The private core}

The following core notion was introduced in Yannelis (1991, Definition 3.1.1, p. 187). It was subsequently used by Allen (1991) who refers to it as the private information core, or the publicly predictable information core. Definition 3.1. An allocation $x: \Omega \rightarrow \prod_{i=1}^{n} X_{i}$ is a private core allocation for $\mathscr{E}$, if the
following conditions hold:

(i) each $x_{i}$ is $\mathscr{F}_{i}$-measurable;

(ii) $\sum_{i=1}^{n} x_{i}(\omega)=\sum_{i=1}^{n} e_{i}(\omega) \mu$-a.e.;

(iii) it is not true that there exist $S \subset\{1,2, \ldots, n\}$ and $y: \Omega \rightarrow \prod_{i \in S} X_{i}$, such that each $y_{i}$ is $\mathscr{F}_{i}$-measurable for all $i \in S, \sum_{i \in S} y_{i}(\omega)=\sum_{i \in S} e_{i}(\omega) \mu-$ a.e. and $\int u_{i}\left(y_{i}(\omega)\right) d \mu(\omega)>$ $\int u_{i}\left(x_{i}(\omega)\right) d \mu(\omega)$ for all $i \in S$.

Condition (i) implies that the net trade of each agent, i.e., $x_{i}-e_{i}$ is $\mathscr{F}_{i}$-measurable (recall that each $e_{i}$ is $\mathscr{F}_{i}$-measurable) and consequently each agent knows his/her own net trade realization. Condition (ii) says that the markets are cleared for almost all states of nature. Note that since $\sum_{i=1}^{n}\left(x_{i}(\omega)-e_{i}(\omega)\right)=0 \mu-a . e$. and by (i) each $x_{i}-e_{i}$ is $\mathscr{F}_{i}$-measurable, it follows that $\sum_{i=1}^{n}\left(x_{i}(\cdot)-e_{i}(\cdot)\right)$ is $\bigvee_{i=1}^{n} \mathscr{F}_{i}$-measurable and therefore, the grand coalition knows the aggregate net trade realization.

Condition (iii) says that it is not possible for a coalition of agents to get together, redistribute their resources among themselves (while each agent in the coalition use his/her own private information) and make the expected utility of each agent in the coalition better off.

It should be noted that the measurability assumptions in (i) and (iii) are equivalent to the fact that:

(i') Each $x_{i}-e_{i}$ is $\mathscr{F}_{i}$-measurable, and

(ii') It is not true that there exist $S$ and $y: \Omega \rightarrow \prod_{i \in S} X_{i}$, such that $y_{i}-e_{i}$ is 
$\mathscr{F}_{i}$-measurable for all $i \in S, \sum_{i \in S} y_{i}(\omega)=\sum_{i \in S} e_{i}(\omega) \mu$-a.e. and $\int u_{i}\left(y_{i}\right) d \mu>\int u_{i}\left(x_{i}\right) d \mu$ for all $i \in S$.

Pick now arbitrarily an agent $j$ in the coalition $S$. Note that since in (iii) above, $\sum_{i \in S}\left(y_{i}(\cdot)-e_{i}(\cdot)\right)=0$, by rearranging we have that $e_{j}(\cdot)-y_{j}(\cdot)=\sum_{i \in S \backslash \backslash j}\left(y_{i}(\cdot)-e_{i}(\cdot)\right)$. Since $e_{j}-y_{j}$ is $\mathscr{F}_{j}$-measurable and $\sum_{i \in S \backslash\{j\}}\left(y_{i}-e_{i}\right)$ is $\bigvee_{i \in S \backslash\{j\}} \mathscr{F}_{i}$-measurable, it is always the case that within a coalition say $S$ the $|S-1|$ members of the coalition can pool their private information and verify the net trade of the remaining agent. ${ }^{4}$

The following theorem proved in Yannelis (1991) provides sufficient conditions which guarantee the existence of a private core allocation for $\mathscr{E}$. The commodity space $Y$ can be the positive cone of any Banach lattice with an order continuous norm.

Theorem 3.1. Let $\mathscr{E}=\left\{\left(X_{i}, u_{i}, e_{i}, \mathscr{F}_{i}, \mu\right): i=1,2, \ldots, n\right\}$ be an exchange economy with differential information, satisfying the following assumptions for each $i(i=1,2, \ldots, n)$ :

(a.3.1) $X_{i}: \Omega \rightarrow 2^{Y}$ is a convex, closed, nonempty valued correspondence;

(a.3.2) $u_{i}: Y \rightarrow \mathbb{R}$ is continuous, integrably bounded and concave.

Then a private core allocation exists in $\mathscr{E}$.

A few technical remarks are in order. Note that in Theorem 3.1 of Yannelis (1991), the set-valued function $X_{i}: \Omega \rightarrow 2^{Y}$ is assumed to be integrably bounded and $\mathscr{F}_{i}$-measurable as well. The latter assumption was needed to show [see Yannelis (1991, p. 191)] that the set $L_{X_{i}}$ which is defined to be a set of all Bochner integrable and $\mathscr{F}_{i}$-measurable selections from the set-valued function $X_{i}: \Omega \rightarrow 2^{Y}$, is nonempty. However, since $e_{i}: \Omega \rightarrow Y$ is Bochner integrable and $\mathscr{F}_{i}$-measurable, we can conclude that $e_{i} \in L_{X_{i}}$ and hence the set $L_{X_{i}}$ is indeed nonempty. This change in assumptions allows us to relax the separability assumption on the commodity space. [Recall that the separability assumption in Yannelis (1991) was needed in one step only, in particular it was used to make the Aumann measurable selection theorem applicable and to show that $L_{X_{i}}$ is nonempty.] Finally, the utility function of each agent, $u_{i}: Y \rightarrow \mathbb{R}$ can only be assumed to be norm continuous instead of weakly continuous as it was assumed in Yannelis (1991). In particular, by virtue of the Lebegue Dominated Convergence Theorem one can show that $\int u_{i} d \mu$ is norm continuous. In view of the concavity of $u_{i}$ it follows from Mazur's Theorem that $\int u_{i} d \mu$ is.also weakly-upper semicontinuous (w-u.s.c.) and the existence proof in Yannelis (1991) remains unchanged. Note that in this case it doesn't make any difference whether we assume that each $\mathscr{F}_{i}$ is a partition, or a sub- $\sigma$-algebra of $\Omega$. Moreover, the dual space of $Y$ doesn't need to have the Radon-Nikodym Property (RNP). For more details on these issues see Balder-Yannelis (1991) or Page (1992).

\footnotetext{
${ }^{4}$ In view of this property of the private core, Allen (1991) refers to it as the publicly predictable information core.
} 


\subsection{The coarse core}

The following definition of a coarse core allocation is taken from Yannelis (1991, p. 187). It is a variant of the coarse core concept first introduced by Wilson (1978) [see also Kobayashi (1980)].

Definition 3.2. An allocation $x: \Omega \rightarrow \prod_{i=1}^{n} X_{i}$ is a coarse core allocation for $\mathscr{E}$, if the following conditions hold:

(i) Each $x_{i}$ is $\mathscr{F}_{i}$-measurable;

(ii) $\sum_{i=1}^{n} x_{i}(\omega)=\sum_{i=1}^{n} e_{i}(\omega) \mu-$ a.e.;

(iii) It is not true that there exist $S \subset\{1,2, \ldots, n\}$ and $y: \Omega \rightarrow \prod_{i \in S} X_{i}$ such that $y_{i}-e_{i}$ is $\bigwedge_{i \in S} \mathscr{F}_{i}$-measurable for all $i \in S, \sum_{i \in S} y_{i}(\omega)=\sum_{i \in S} e_{i}(\omega) \mu-$ a.e. and $\int u_{i}\left(y_{i}(\omega)\right)$. $d \mu(\omega)>\int u_{i}\left(x_{i}(\omega)\right) d \mu(\omega)$ for all $i \in S$.

Conditions (i) and (ii) are discussed above. Condition (iii) says that it is not possible for a coalition of agents by redistributing their initial endowments (based on information which is common knowledge to the coalition) to make the expected utility of each agent in the coalition better off.

We now state a result on the existence of coarse core allocations that follows directly from Theorem 3.1 in Yannelis (1991), simply by noticing that the set of all private core allocations for $\mathscr{E}$ is a strict subset of the set of all coarse core allocations for $\mathscr{E}$.

Theorem 3.2. Let $\mathscr{E}=\left\{\left(X_{i}, u_{i}, e_{i}, \mathscr{F}_{i}, \mu\right): i=1,2, \ldots, n\right\}$ be an exchange economy with differential information satisfying for each $i,(i=1,2, \ldots, n)$ all the assumptions of Theorem 3.1 above. Then a coarse core allocation exists in $\mathscr{E}$.

Note that if condition (i) in Definition 3.2 is replaced by:

(i') Each $x_{i}$ is $\bigwedge_{i \in I} \mathscr{F}_{i}$-measurable,

then we will indicate in Sect. 5 that such a coarse core notion which we will call a strong coarse core allocation for $\mathscr{E}$, may not exist. In particular, we show in Sect. 5 that there exist private information exchange economies satisfying all the assumptions of Theorem 3.2, but for which strong coarse core allocations may not exist. Note that what we call here a "strong coarse core" corresponds to the "coarse core" in Allen (1991). It is exactly for this reason that Allen (1991) concludes that the strong coarse core may be empty.

\subsection{The fine core}

The following core notion is taken from Yannelis (1991, p. 188) and is a variant of the fine core concept introduced by Wilson (1978). Definition 3.3. An allocation $x: \Omega \rightarrow \prod_{i=1}^{n} X_{i}$ is a fine core allocation for $\mathscr{E}$ if the
following conditions hold:

(i) Each $x_{i}$ is $\mathscr{F}_{i}$-measurable.

(ii) $\sum_{i=1}^{n} x_{i}(\omega)=\sum_{i=1}^{n} e_{i}(\omega) \mu-$ a.e. 
(iii) It is not true that there exist $S \subset\{1,2, \ldots, n\}$ and $y: \Omega \rightarrow \prod_{i \in S} X_{i}$, such that $y_{i}-e_{i}$ is $\underset{i \in S}{\bigvee} \mathscr{F}_{i}$-measurable for all $i \in S, \sum_{i \in S} y_{i}(\omega)=\sum_{i \in S} e_{i}(\omega) \mu-$ a.e. and $\int u_{i}\left(y_{i}(\omega)\right) d \mu(\omega)$ $>\int u_{i}\left(x_{i}(\omega)\right) d \mu(\omega)$ for all $i \in S$.

Since conditions (i) and (ii) are the same with those in Definition 3.1 we only need to interpret condition (iii). The latter condition says that no coalition of agents can redistribute their own initial endowment using their pooled information and make every member in the coalition better off. Formally, since each net trade $y_{i}-e_{i}$ is $\bigvee_{i \in S} \mathscr{F}_{i}$-measurable for each $i \in S$, we can conclude that net trades are now based on the pooled information of the coalition. It was remarked in Yannelis (1991, p. 188) that the above fine core may be empty. ${ }^{5}$ In Sect. 5 we show by means of an example (which satisfies all the assumptions of Theorem 3.1) that indeed the fine core may be empty.

If condition (i) in Definition 3.3 is replaced by:

(i') Each $x_{i}$ is $\bigvee_{i=1}^{n} \mathscr{F}_{i}$-measurable,

then we will call such a core notion a weak fine core allocation for $\mathscr{E}^{6}$

The theorem below indicates that a weak fine core allocation exists in $\mathscr{E}$.

Theorem 3.3. Let $\mathscr{E}=\left\{\left(X_{i}, u_{i}, e_{i}, \mathscr{F}_{i}, \mu\right): i=1,2, \ldots, n\right\}$ be an exchange economy with differential information satisfying for each $i(i=1,2, \ldots, n)$ all the assumptions of Theorem 3.1. Then a weak fine core allocation exists in $\mathscr{E}$.

Proof. It follows directly from Theorem 3.1 as follows. For $S \subset\{1,2, \ldots, n\}$ denote $\bigvee_{i \in S} \mathscr{F}_{i}$ by $\mathscr{F}^{S}$. Define $L_{X_{i}}$ as: $L_{X_{i}}=\left\{x_{i}: x_{i}: \Omega \rightarrow Y\right.$ is Bochner integrable, $\underset{s \in I}{\bigvee}$ $\mathscr{F}^{S}$-measurable and $x_{i}(\omega) \in X_{i}(\omega) \mu$-a.e. $\}$. By Theorem 3.1 there exists an $x \in \prod_{i=1}^{n} L_{X_{i}}$, such that:

(i) $\sum_{i=1}^{n} x_{i}(\omega)=\sum_{i=1}^{n} e_{i}(\omega) \mu$-a.e.

(ii) It is not true that there exist $S$ and $\left(y_{i}\right)_{i \in S} \in \prod_{i \in S} L_{x_{i}}$ such that $\sum_{i \in S} y_{i}(\omega)=$ $\sum_{i \in S} e_{i}(\omega) \mu$-a.e. and $\int u_{i}\left(y_{i}\right) d \mu>\int \mu_{i}\left(x_{i}\right) d \mu$ for all $i \in S$.

Observe that since $x \in \prod_{i=1}^{n} L_{X_{i}}$ each $x_{i}$ is $\bigvee_{i=1}^{n} \mathscr{F}_{i}$-measurable. (ii) implies that condition

\footnotetext{
${ }^{5}$ Wilson (1978) has already shown by means of an example that his fine core notion may be empty. However, his example is not entirely consistent with the above notion. Recall that in the Wilson setting allocations and endowments are not necessarily measurable. In a public finance setting Berliant (1992) has also shown that a fine core-type notion may not exist.

${ }^{6}$ Clearly the set of all fine core allocations for $\mathscr{E}$ is a strict subset of the set of weak fine core allocations for $\mathscr{E}$. Also, notice that in the definitions of the fine, coarse, and weak fine core the measurability of the final allocation is equivalent to the measurability of the net trades. However, this is not the case for the strong coarse core.

${ }^{7}$ Note that $y_{i}-e_{i}, \bigvee_{i \in S} \mathscr{F}_{i}$-measurable for all $i \in S$ is equivalent (recall that $e_{i}$ is $\mathscr{F}_{i}$-measurable) to the fact that $y_{i}$ is $\underset{i \in S}{\vee} \mathscr{F}_{i}$-measurable for all $i \in S$.
} 
(iii) of Definition 3.3 holds. To see this, suppose otherwise than there exist $S \subset I$ and $y: \Omega \rightarrow \prod_{i \in S} X_{i}$, such that $y_{i}-e_{i}$ is $\bigvee_{i \in S} \mathscr{F}_{i}$-measurable ${ }^{7}$ for all $i \in S, \sum_{i \in S} y_{i}(\omega)=\sum_{i \in S} e_{i}(\omega)$ $\mu-a . e$. and $\int u_{i}\left(y_{i}\right) d \mu>\int u_{i}\left(x_{i}\right) d \mu$ for all $i \in S$. Since $y_{i}$ is $\bigvee_{i \in S} \underset{\mathscr{F}}{i \in S}$-measurable it is also $\underset{S \subset I}{\bigvee} \mathscr{F}^{S}$-measurable and therefore $y_{i} \in L_{X_{i}}$ for all $i \in S$, a contradiction to condition (ii) above. Hence, we conclude that $x: \Omega \rightarrow \prod_{i=1}^{n} X_{i}$ is a weak fine core
allocation for $\mathscr{E}$ and this completes the proof.

It should be noted that our definition of a weak fine core allocation for $\mathscr{E}$ corresponds to Allen's (1991) fine core allocation for $\mathscr{E}$, who has proved a less general version of Theorem 3.3 .

\section{Truthful revelation of information in the core}

One of the basic questions that one may ask is whether the core notions defined in the previous section are coalitionally incentive compatible. That is, is there truthful revelation of information in each coalition? We show below that indeed the private, the coarse, and the fine cores are incentive compatible. We define rigorously below a notion of incentive compatibility which was introduced in Krasa-Yannelis (1991).

Definition 4.1. A feasible allocation is said to be coalitionally incentive compatible if and only if the following does not hold: There exists coalition $S \subset I$ and two states $a$ and $b$ that members of $I / S$ cannot distinguish (i.e., $a$ and $b$ are in the same event of the partition for every agent not in the coalition $S$ ) and such that members of $S$ are better off by announcing $b$ whenever $a$ has actually occurred. Formally, the feasible allocation $x: \Omega \rightarrow \prod_{i=1}^{n} X_{i}$ is said to be coalitional incentive compatible for $\mathscr{E}$ if it is not true that we can find a coalition $S$ and states $a, b$ with $a \in E_{i}(b)$ for every $i \notin S$, such that $u_{i}\left(e_{i}(a)+x_{i}(b)-e_{i}(b)\right)>u_{i}\left(x_{i}(a)\right)$ for all $i \in S$.

It turns out that in the case of one commodity per state, if preferences are monotone then the $\mathscr{F}_{i}$-measurability of a feasible allocation implies that the allocation is also coalitionally incentive compatible. For the result below for each $i, X_{i}$ is a set-valued function from $\Omega$ to $\mathbb{R}_{+}$, i.e., there is only one good per state.

Proposition 4.1. Let $x: \Omega \rightarrow \prod_{i=1}^{n} X_{i}$ be a feasible allocation for $\mathscr{E}$. Suppose that:

(i) Each $x_{i}$ is $\mathscr{F}_{i}$-measurable.

(ii) For any $y, z$ in $\mathbb{R}_{+}$and for each $i \in I$, if $y>z$ then $u_{i}(y)>u_{i}(z)$ (monotonicity). Then the allocation $x$ is coalitionally incentive compatible.

Proof. Suppose otherwise, then there exist $S \subset I$ and $a, b, a \in E_{i}(b)$ for all $i \notin S$ such that

$$
u_{i}\left(e_{i}(a)+x_{i}(b)-e_{i}(b)\right)>u_{i}\left(x_{i}(a)\right) \text { for all } i \in S .
$$

Since $x$ is feasible it follows that

$$
\sum_{i \in S}\left(x_{i}(\cdot)-e_{i}(\cdot)\right)=\sum_{i \notin S}\left(x_{i}(\cdot)-e_{i}(\cdot)\right) .
$$


Since by definition the initial endowment of each agent is $\mathscr{F}_{i}$-measurable and by assumption (i) each $x_{i}$ is $\mathscr{F}_{i}$-measurable, it follows that $x_{i}-e_{i}$ is $\mathscr{F}_{i}$-measurable and consequently we can conclude that for any coalition $T \subset I$, the sum $\sum_{i \in T}\left(x_{i}(\cdot)-e_{i}(\cdot)\right)$ is $\underset{i \in T}{\vee} \mathscr{F}_{i}$-measurable. Since $a \in E_{i}(b)$ for every $i \notin S$ it follows that $a \in \bigcap_{i \notin S} E_{i}(b)$. Clearly $\bigcap_{i \notin S}^{i \in T} E_{i}(b)$ is an element of $\bigvee_{i \notin S} \mathscr{F}_{i}$. By the above reasoning the $\operatorname{sum} \sum_{i \notin S}^{i \notin S}\left(x_{i}(\cdot)-e_{i}(\cdot)\right)$ iфs is $\underset{i \notin S}{V} \mathscr{F}_{i}$-measurable and therefore we can conclude that

$$
\sum_{j \notin S}\left(x_{i}(a)-e_{i}(a)\right)=\sum_{i \notin S}\left(x_{i}(b)-e_{i}(b)\right) .
$$

Hence,

$$
\begin{aligned}
\sum_{i \in S}\left(x_{i}(a)-e_{i}(a)\right) & =-\sum_{i \notin S}\left(x_{i}(a)-e_{i}(a)\right) \\
& =-\sum_{i \notin S}\left(x_{i}(b)-e_{i}(b)\right) \quad(\text { recall }(2)) \\
& =\sum_{i \in S}\left(x_{i}(b)-e_{i}(b)\right) .
\end{aligned}
$$

We now show that $x_{i}(a)-e_{i}(a)=x_{i}(b)-e_{i}(b)$ for all $i \in S$. Suppose otherwise, i.e., $x_{i}(a)-e_{i}(a) \neq x_{i}(b)-e_{i}(b)$ for some $i \in S$. Without loss of generality we may assume that $x_{i}(b)-e_{i}(b)>x_{i}(a)-e_{i}(a)$ for some $i \in S$. It follows from (3) that $x_{j}(a)-e_{j}(a)>$ $x_{j}(b)-e_{j}(b)$ for some agent $j \in S$. Since $x_{j}(a)>x_{j}(b)-e_{j}(b)+e_{j}(a)$ it follows from assumption (ii) that $u_{j}\left(x_{j}(a)\right)>u_{j}\left(x_{j}(b)-e_{j}(b)+e_{j}(a)\right)$ for some $j \in S$, a contradiction to (1). Hence, we conclude that $x_{i}(a)-e_{i}(a)=x_{i}(b)-e_{i}(b)$ for all $i \in S$. But then $u_{i}\left(e_{i}(a)+x_{i}(b)-e_{i}(b)\right)=u_{i}\left(e_{i}(a)+x_{i}(a)-e_{i}(a)\right)=u_{i}\left(x_{i}(a)\right)$ for all $i \in S$, a contradiction to (1). This completes the proof of the proposition.

It follows now directly from Proposition 4.1 that any private, coarse, or fine core allocation of the one commodity per state economy $\mathscr{E}$ is coalitionally incentive compatible provided that preferences are monotone. The following Corollary of Proposition 4.1 holds:

Corollary 4.1. Let $\mathscr{E}=\left\{\left(X_{i}, u_{i}, e_{i}, \mathscr{F}_{i}, \mu\right): i=1,2, \ldots, n\right\}$ be an exchange economy with differential information satisfying assumption (ii) of Theorem 4.1. If $x: \Omega \rightarrow \prod_{i=1}^{n} X_{i}$ is either a private, a coarse or a fine core allocation for $\mathscr{E}$, then $x$ is coalitionally incentive compatible.

We focus now on the private core of $\mathscr{E}$ and show that it is always coalitionally incentive compatible provided that preferences are monotone. The one good per state assumption is now dropped. Before stating the main result of this section, we modify Definition 4.1 to permit for more than one good per state.

Definition 4.2. A feasible allocation $x: \Omega \rightarrow \prod_{i=1}^{n} X_{i}$ is said to be weak coalitionally incentive compatible for $\mathscr{E}$, if it is not true that there exist coalition $S$ and states $a, b$ in $\Omega$ such that:

(i) $E_{i}(a) \in \bigwedge_{i \in S} \mathscr{F}_{i}, \mu\left(E_{i}(a)\right)>0$, 
(ii) $a \in E_{i}(b)$ for $i \notin S$, and

(iii) $u_{i}\left(e_{i}(a)+x_{i}(b)-e_{i}(b)\right)>u_{i}\left(x_{i}(a)\right)$ for all $i \in S$.

This notion of incentive compatibility states that it is not possible for any coalition $S$ to become better off by announcing a false state, which agents not in the coalition $S$ cannot distinguish from the true state. Conditions (ii) and (iii) are the same as in Definition 4.1. Condition (i) says that the members of the coalition $S$ should agree on whether $a$ state has occurred. In other words, the event containing the realized (misreported) state $a$, i.e., $E_{i}(a)$ is known to every member of the coalition. Thus, there is no "double cross" among the members of a coalition that they agree to lie. The condition $\mu\left(E_{i}(a)\right)>0$ shows that there is a non-negligible possibility for misreporting.

We are now ready to state the main result of this section:

Theorem 4.1. Let $\mathscr{E}=\left\{\left(X_{i}, u_{i}, e_{i}, \mathscr{F}_{i}, \mu\right): i=1,2, \ldots, n\right\}$ be an exchange economy with differential information satisfying for each $i(i=1,2, \ldots, n)$, all the assumptions of Theorem 3.1. Moreover, suppose that preferences are monotone. Then any private core allocation for $\mathscr{E}$ is weak coalitionally incentive compatible.

Proof. Let $x$ be a private core allocation for $\mathscr{E}$. For each $i$, define $z_{i}: \Omega \rightarrow X_{i}$ by $z_{i}(\cdot)=x_{i}(\cdot)-e_{i}(\cdot)$.

Suppose that $x$ is not weakly coalitionally incentive compatible. Then there exist $S \subset I$ and $a, b \in \Omega$ such that:

(i) $E_{i}(a) \in \bigwedge_{i \in S} \mathscr{F}_{i}, \mu\left(E_{i}(a)\right)>0$,

(ii) $a \in E_{i}(b), i \notin S$, and

(iii) $u_{i}\left(e_{i}(a)+z_{i}(b)\right)>u_{i}\left(x_{i}(a)\right)$ for all $i \in S$.

First notice that since $e_{i}(\cdot)$ and $x_{i}(\cdot)$ are $\mathscr{F}_{i}$-measurable, (iii) implies that for all $i \in S$, $u_{i}\left(e_{i}(t)+z_{i}(b)\right)>u_{i}\left(x_{i}(t)\right)$ for all $t \in E_{i}(a)$.

Since $a \in E_{i}(b)$ for all $i \notin S$ we have that $a \in \bigcap_{i \notin S} E_{i}(b)$. Clearly $\bigcap_{i \notin S} E_{i}(b) \in \bigvee_{i \notin S} \mathscr{F}_{i}$.

We know that $\sum_{i \notin S} z_{i}(\cdot)$ is $\bigvee_{i \notin S} \mathscr{F}_{i}$-measurable and since $a \in \bigcap_{i \notin S}^{i \notin S} E_{i}(b)$ we conclude that $\sum_{i \notin S} z_{i}(a)=\sum_{i \notin S} z_{i}(b)$. By the feasibility of $x$ we have that $\sum_{i \in S}^{i \notin S} z_{i}(a)=-\sum_{i \notin S} z_{i}(a)$ and thus

$$
\sum_{i \in S} z_{i}(a)=\sum_{i \in S} z_{i}(b)
$$

Consider now the following net trades: ${ }^{8}$

$$
z_{i}^{*}(t)=z_{i}(t) \chi_{\Omega \backslash E_{i}(a)}+z_{i}(b) \chi_{E_{i}(a)} \text { for } i \in S .
$$

The above net trades are $\mathscr{F}_{i}$-measurable (since each $z_{i}$ is $\mathscr{F}_{i}$-measurable) and feasible. Indeed, since $E_{i}(a) \in \bigvee \mathscr{F}_{i}$ it follows that for $t \in E_{i}(a), z_{i}(t)=z_{i}(a)$ for all $i \in S$. Hence, if $t \notin E_{i}(a), \sum_{i \in S} z_{i}^{*}(t)+\sum_{i \notin S} z_{i}(t)=\sum_{i \in I} z_{i}(t)=0$ (recall the feasibility of $x$ ).

${ }^{8}$ The symbol $\chi$ below denotes the characteristic function. See also Section 7 . 
If $t \in E_{i}(a), \sum_{i \in S} z_{i}^{*}(t)+\sum_{i \notin S} z_{i}(t)=\sum_{i \in S} z_{i}(b)+\sum_{i \notin S} z_{i}(t)=\sum_{i \in S} z_{i}(b)-\sum_{i \in S} z_{i}(t)=\sum_{i \in S} z_{i}(b)-$ $\sum_{i \in S} z_{i}(a)=0$ (by $\left(\begin{array}{l}3) \\ )\end{array}\right)$. We can now construct the following allocation. For each $i$, $(i=1,2, \ldots, n)$ let

$$
x_{i}^{*}(\cdot)=\left\{\begin{array}{l}
e_{i}(\cdot)+z_{i}^{*}(\cdot), \quad i \in S \\
e_{i}(\cdot)+z_{i}(\cdot), \quad i \notin S
\end{array}\right.
$$

Notice that for all $i \in S, u_{i}\left(x_{i}^{*}(t)\right)=u_{i}\left(e_{i}(t)+z_{i}(b)\right)>u_{i}\left(x_{i}(t)\right)$ for $\left(t \in E_{i}(a)\right)$. Since $\mu\left(E_{i}(a)\right)>0$, we have that

$$
\begin{aligned}
& \int u_{i}\left(x_{i}^{*}(t)\right) d \mu(t)>\int u_{i}\left(x_{i}(t)\right) d \mu(t) \text { for all } i \in S, \text { and } \\
& \int u_{i}\left(x_{i}^{*}(t)\right) d \mu(t)=\int u_{i}\left(x_{i}(t)\right) d \mu(t) \text { for all } i \notin S .
\end{aligned}
$$

Since $\int u_{i}(\cdot)$ is norm-continuous [by virtue of the Lebegue Dominated Convergence Theorem], by choosing $A \in \bigwedge_{i \in I} \mathscr{F}_{i}$ with $\mu(A)>0$, we can find a function $\varepsilon \cdot \chi_{A}$, where $\varepsilon \geqq 0$, such that for $\varepsilon$ sufficiently small, $\left\|\varepsilon \chi_{A}\right\|<\delta$ so that

$$
\int u_{i}\left(x_{i}^{*}-\varepsilon \chi_{A}\right) d \mu>\int u_{i}\left(x_{i}\right) d \mu \text { for all } i \in S .
$$
By monotonicity of preferences for $i \notin S, u_{i}\left(x_{i}^{*}(t)+\frac{1}{|I / S|} \varepsilon \cdot \chi_{A}\right)>u_{i}\left(x_{i}(t)\right)$ for $t \in A$.
Since $\mu(A)>0$ we have that:

$$
\int u_{i}\left(x_{i}^{*}+\frac{1}{|I / S|} \varepsilon \chi_{A}\right) d \mu>\int u_{i}\left(x_{i}\right) d \mu \text { for all } i \notin S .
$$

Hence, the allocation:

$$
x_{i}^{* *}= \begin{cases}x_{i}^{*}-\varepsilon \chi_{A}, & i \in S \\ x_{i}^{*}+\frac{1}{|I / S|} \varepsilon \chi_{A}, & i \notin S\end{cases}
$$

is $\mathscr{F}_{i}$-measurable (since it is $x_{i}^{*}$ perturbed over a measurable set), it is feasible for the grand coalition and it follows from (5) and (6) that $\int u_{i}\left(x^{* *}\right) d \mu>\int u_{i}(x) d \mu$ for all $i,(i=1,2, \ldots, n)$, a contradiction to the fact that $x$ has been assumed to be a private core allocation for $\mathscr{E}$. This completes the proof of the theorem.

Remark 4.1. Although the theorem says that any private core allocation is weakly coalitionally incentive compatible, the proof shows that a stronger result is true, i.e., any private Pareto optimal allocation [see Yannelis (1991), Definition 3.1.2, p. 188] will be weakly coalitionally incentive compatible as well.

\section{Interpretation of the private coarse and fine core allocations}

In an economy with differential information it is reasonable to expect that an agent with even a zero initial endowment but better (finer) private information than all other agents that matters to the rest of the agents, should be able to exchange his/her superior private information for actual goods. Obviously, this is not the case if we adopt as an equilibrium notion the traditional Walrasian equilibrium 
(i.e., any rational expectations equilibrium notion). In particular, in the Walrasian equilibrium if an agent has no initial endowment, even if his/her information is better and essential to all the other agents, he/she always ends up with zero consumption. (To see this simply note that in any Walrasian equilibrium notion this agent will have to maximize his/her expected utility conditional on his/her own private information, subject to a budget set which is zero.) We believe that for an equilibrium notion to be suitable in a differential information economy framework it should be able to reward an agent with superior information provided that the information matters to the rest of the agents (even if this agent has no endowment of physical good). The example below demonstrates that this is the case for the private core and the coarse core, but not for the fine core.

Example 5.1: Consider an economy with three agents denoted by $J, K, L$ and three states of nature denoted by $a, b, c$. There is only one good in each state. The random initial endowments of the agents are given as follows: Agent $J$ 's is $(10,10,0)$, agent $K$ 's is $(10,0,10)$ and agent $L$ 's is $(0,0,0)$. Their private information sets are: $\mathscr{F}_{J}=\{\{a, b\},\{c\}\}, \mathscr{F}_{K}=\{\{a, c\},\{b\}\}$ and $\mathscr{F}_{L}=\{\{a\},\{b, c\}\}$. All agents have the same utility function given by $\sqrt{x}$ and each state occurs with the same probability. We first analyse the private core.

\subsection{The private core}

The above example satisfies all the assumptions of Theorem 3.1 and therefore a private information core allocation exists in this three-person exchange economy with differential information. We show that in a private core allocation agent $L$ will have positive consumption. First note that since the net trade of each agent must be $\mathscr{F}_{i}$-measurable, $J$ and $K$ together cannot make any beneficial trades, i.e., the only trades possible between $J$ and $K$ are state independent and these trades do not make them better off. However, the participation of agent $L$ in the economy makes everybody better off. In fact it can be easily checked that the following allocation

$$
x_{i}^{*}=\left\{\begin{array}{lll}
(8,8,2) & \text { for } & i=J \\
(8,2,8) & \text { for } i=K \\
(4,0,0) & \text { for } i=L,
\end{array}\right.
$$

is a private core allocation, i.e., $x_{i}^{*}$ is $\mathscr{F}_{i}$-measurable, feasible and it cannot be dominated by any coalition.

In this example, agents $J$ and $K$ cannot undertake any risk sharing without agent $L$. Since agent $L$ has superior information he/she acts as an intermediary who executes the correct trades and as a consequence gets rewarded for this service. All three agents are better off after trade has taken place (simply note that $x_{i}^{*}$ for $i=J, K, L$ is individually rational, i.e., $\left.\int u_{i}\left(x^{*}\right) d \mu \geqq \int u_{i}(e) d \mu\right)$. In sharp contrast with the core notion, if we had adopted the Walrasian equilibrium, then agent $L$ would have obtained zero consumption since he/she started with zero initial endowments. This holds no matter whether or not agent $L$ 's information is useful to the other agents.

If the private information of agent $L$ were not useful to agents $J$ and $K$ then agent $L$ would have obtained zero consumption. For instance if the private 
information set of agent $L$ is $\mathscr{F}_{L}=\{a, b, c\}$ then the initial endowment is the unique private core allocation. This result is quite interesting because our example indicates that the private core takes into account the information advantage or superiority of an individual in an explicit way. It is exactly for this reason that we believe that the private core serves to provide more plausible outcomes than the Walrasian equilibrium.

\subsection{Coarse core}

Let us now examine the coarse core allocation. We know that any private core allocation is also a coarse core allocation. Hence the private core allocation (5.1) is a coarse core allocation as well. ${ }^{9}$ To show that the coarse core allocation takes into account the superiority or information advantage of an agent, simply observe that if agent $L$ 's private information in Example 5.1 were the trivial partition, i.e., $\mathscr{F}_{L}=\{a, b, c\}$, then the initial endowment is the only coarse core allocation, where agent $L$ receives zero in all states.

The strong coarse core in this example is empty. Simply note that the strong coarse core allocation must be $\left(\mathscr{F}_{J} \wedge \mathscr{F}_{K} \wedge \mathscr{F}_{L}\right)$-measurable, but the meet of these three partitions is the trivial partition, i.e., $\mathscr{F}_{J} \wedge \mathscr{F}_{K} \wedge \mathscr{F}_{L}=\{a, b, c\}$. This implies that the consumption of each agent must be the same in each state. However, given the structure of the initial endowments it is easily seen that no feasible allocation can give to each agent the same consumption in all states and dominate the initial endowments. Moreover the initial endowment state is not a strong coarse allocation since it is not $\left(\mathscr{F}_{J} \wedge \mathscr{F}_{K} \wedge \mathscr{F}_{L}\right)$-measurable. Hence the strong coarse core is empty in this example.

\subsection{Fine core}

We now show that the fine core is empty in Example 5.1. To see this, note that any ${ }^{10} \mathscr{F}_{i}$-measurable allocation $(i=J, K, L)$ which is beneficial to agents $J$ and $K$ can be achieved only through agent $L$ and this agent ends up with positive consumption (e.g., the allocation in (5.1)) in state $\{a\}$. Since improvements for agents $J$ and $K$ can be made with $\left(\mathscr{F}_{J} \vee \mathscr{F}_{K}\right)$-measurable allocations and $\{a\}$ belongs to $\mathscr{F}_{J} \vee \mathscr{F}_{K}=\{\{a\},\{b\},\{c\}\}$, it follows that all $\mathscr{F}_{i}$-measurable $(i=J, K, L)$ allocations are blocked by the coalition $\{J, K\}$ which in turn can share agent $L$ 's consumption in state $\{a\}$, e.g., the allocation in (5.1) can be dominated by the following allocation:

$$
y_{i}^{*}=\left\{\begin{aligned}
(10,8,2) & \text { for } i=J \\
(10,2,8) & \text { for } i=K \\
(0,0,0) & \text { for } i=L .
\end{aligned}\right.
$$

Hence, we can conclude that the fine core is empty.

\footnotetext{
${ }^{9}$ In fact it can be shown that in this example all $\mathscr{F}_{i}$-measurable $(i=J, K, L)$ allocations $x$ which are individually rational (i.e., $\left.\int u(x) d \mu \geqq \int u(e) d \mu\right)$ constitute the coarse core.

${ }^{10}$ Except from the initial endowment which is dominated by the allocation in (5.1).
} 
However, the weak fine core exists. It can be easily checked that the allocation

$$
y_{i}= \begin{cases}(10,5,5) & \text { for } i=J, K \\ (0,0,0) & \text { for } i=L\end{cases}
$$

is in the weak fine core. However, it is not incentive compatible. Simply note that if state $a$ occurs then agent $J$ can become better off by reporting state $c$ and the latter state is not distinguishable from a by agent $K$. In particular, $u_{J}\left(e_{J}(a)+x_{j}(c)-\right.$ $\left.e_{J}(c)\right)=u_{J}(10+5)>u_{J}\left(x_{J}(a)\right)=u_{J}(10)$. Using the same reasoning the reader can verify that agent $K$ can become better off by reporting state $b$ whenever state $a$ occurs.

In contrast with the private core, the information superiority of agent $L$ is not taken into account by the fine core. Indeed, if agent $L$ 's partition is either $\mathscr{F}_{L}=\{a, b, c\}$ or $\mathscr{F}_{L}=\{\{a\},\{b\},\{c\}\}$ the above weak fine core allocation remains unchanged (compare with the private core). This result should not be surprising since whenever agents in a coalition pool their own information, any informational advantage that an agent may have disappears.

\subsection{The unequal treatment of the private core}

In Example 5.1 the agent with zero initial endowments and superior information useful to the rest of the economy facilitated the trades, i.e., he/she served as an intermediary. Obviously, by executing the correct trades he/she made all other agents better off (Pareto improvement) and was compensated for this service by consuming some of the goods.

We now provide an example with two intermediaries.

Example 5.4.1. Consider the Example 5.1 with one additional agent $M$ whose initial endowment is zero in each state, he/she has the same utility function with the other three agents, i.e., $\sqrt{x}$ and let his/her private information set be $\mathscr{F}_{M}=\{\{a\},\{b\},\{c\}\}$. (Agent's $J, K, L$ initial endowments and partitions remain the same as in Example 5.1.)

Clearly, the above four-person economy with differential information satisfies all the assumptions of Theorem 3.1 and therefore a private core allocation exists. We will show that agents $L$ and $M$ can serve as intermediaries. One can easily check that the allocation:

$$
x_{i}^{*}= \begin{cases}(8,8,2) & \text { for } \quad i=J \\ (8,2,8) & \text { for } i=K \\ \left(\ell_{1}, 0,0\right) & \text { for } i=L, \ell_{1} \geqq 0 \\ \left(m_{1}, 0,0\right) & \text { for } \quad i=M, m_{1} \geqq 0\end{cases}
$$

where $\ell_{1}+m_{1}=4$, is a private core allocation. Obviously either agent $L$ or $M$ may serve as an intermediary or both may serve simultaneously. Their final allocation in state $a$ depends on the extent that their information was used to carry out the trades. Note that even if agent $M$ has the same partition as agent $L$, i.e., $\mathscr{F}_{M}=\{\{a\},\{b, c\}\}=\mathscr{F}_{L}$, the set of private core allocations remains the same. Hence, one can conclude that there is no equal treatment, i.e., agents with 
identical characteristics (utility function, initial endowment, and private information set) may receive different utility private core allocations. ${ }^{11}$ Notice that the value allocation of an economy with differential information as defined in Krasa-Yannelis (1991) does not have the equal treatment property as well [just endow each agent in the Scafuri-Yannelis (1984) example with the full information partition].

\subsection{Independence of private information sets}

It should be noted that whether trade takes place or not depends crucially on the structure of the information (or the initial endowments) in a private information economy. In particular, we show below that in a differential information economy with one good per state if the private information sets of the agents are independent (a term which is defined below) then trade does not take place. Obviously, in this case there is no need for an intermediary. Hence, we can conclude that a sufficient condition for trade to take place is that the private information sets should not be independent.

We begin with a definition.

Definition 5.2. Let $\mathscr{E}=\left\{\left(X_{i}, u_{i}, e_{i}, \mathscr{F}_{i}, \mu\right): i=1,2, \ldots, n\right\}$ be a private information economy. We say that $\mathscr{F}_{i}$ is independent of $\mathscr{F}_{j}, i \neq j,(i, j=1,2, \ldots, n)$ if $\mu(A \cap B)=$ $\mu(A) \cdot \mu(B)$ for $A \in \mathscr{F}_{i}$ and $B \in \mathscr{F}_{j}$.

It can be easily shown that the above definition implies independence of the initial endowments (recall that each $e_{i}$ is $\mathscr{F}_{i}$-measurable), i.e.,

$$
\int\left[e_{i}(\omega) \cdot e_{j}(\omega)\right] d \mu(\omega)=\int e_{i}(\omega) d \mu(\omega) \cdot \int e_{j}(\omega) d \mu(\omega) \text { for } i \neq j, \quad(i, j=1,2, \ldots, n) .
$$

We are ready now to state the following proposition.

Proposition 5.2. Let $\mathscr{E}$ be an exchange economy with private information satisfying all the assumptions of Theorem 3.1. Moreover, suppose that there is only one good per state, preferences are monotone, and that each $\mathscr{F}_{i}$ is independent of $\mathscr{F}_{j}, i \neq j$. Then the unique private core allocation is the initial endowment.

The proof of this proposition follows directly from the following two Lemmata. Indeed if there is a private information core allocation say $x^{*}(\cdot)=\left(x_{1}^{*}(\cdot), \ldots, x_{n}^{*}(\cdot)\right)$ (other than the initial endowment), then by setting for each $i \in I, z_{i}(\omega)=x_{i}^{*}(\omega)-e_{i}(\omega)$ $\mu$-a.e. and letting $I=S$ in Lemma 5.2 below we can conclude that $\int e_{i}(\omega) d \mu(\omega)>$ $\int x_{i}^{*}(\omega) d \mu(\omega)$, i.e., $x^{*}$ is not individually rational.

Lemma 5.1. Let $\mathscr{E}=\left\{\left(X_{i}, u_{i}, e_{i}, \mathscr{F}_{i}, \mu\right): i=1,2, \ldots, n\right\}$ be an exchange economy with differential information satisfying the assumptions of Proposition 5.2. Consider $a$ coalition $S$ whose members have independent partitions. If for each $i \in S, z_{i}: \Omega \rightarrow X_{i}$ is the net trade of agent $i$ which is $\mathscr{F}_{i}$-measurable and $\sum_{i \in S} z_{i}(\omega)=0 \mu$-a.e., then for each $i \in S$ either $z_{i}(\omega)<0 \mu-$ a.e., or $z_{i}(\omega) \geqq 0 \mu$-a.e.

\footnotetext{
${ }^{11}$ In particular, the final allocation of each intermediary depends on the volume of trades that they carry through.
} 
Proof. Choose an agent $i$ in $S$. Since $\sum_{i \in S} z_{i}(\cdot)=0$ it follows that

$$
z_{i}(\cdot)=-\sum_{\substack{j \neq i \\ j \in S}} z_{j}(\cdot)
$$

Since each $z_{j}$ is $\mathscr{F}_{j}$-measurable it follows that $-\sum_{\substack{j \neq i \\ j \in S}} z_{j}(\cdot)$ is $\underset{\substack{j \neq i \\ j \in S}}{\vee} \mathscr{F}_{i}$-measurable and therefore by virtue of (5.4.1) we can conclude that $z_{i}(\cdot)$ is $\underset{\substack{j \neq i \\ j \in S}}{\bigvee} \mathscr{F}_{j}$-measurable. Since $z_{i}(\cdot)$ is $\underset{\substack{j \neq i \\ j \in S}}{\bigvee} \mathscr{F}_{j}$-measurable the set $z_{i}^{-1}([0, \infty])=\left\{\omega \in \Omega: z_{i}(\omega) \in[0, \infty]\right\}=\{\omega \in \Omega$ : $\left.z_{i}(\omega) \geqq 0\right\}$ belongs to $\mathscr{F}_{i} \cap\left(\bigvee_{\substack{j \neq i \\ j \in S}} \mathscr{F}_{j}\right)$. Since $\mathscr{F}_{i}$ is independent of $\mathscr{F}_{j} i \neq j, i, j$ in $S$, it follows that $\mathscr{F}_{i}$ is independent of $\bigvee_{\substack{j \neq i \\ j \in S}} \mathscr{F}_{j}$. Since $z_{i}^{-1}[0, \infty)$ is $\mathscr{F}_{i} \cap\left(\bigvee_{\substack{j \neq i \\ j \in S}}^{\bigvee \mathscr{F}_{j}}\right)$ measurable, $z_{i}^{-1}[0, \infty)$ is independent to itself. Hence, $\mu\left(z_{i}^{-1}[0, \infty] \cap z_{i}^{-1}([0, \infty])\right)=$ $\mu\left(z_{i}^{-1}[0, \infty]\right) \cdot \mu\left(z_{i}^{-1}[0, \infty]\right)$ and so $\mu\left(z_{i}^{-1}[0, \infty]\right)=\left[\mu\left(z_{i}^{-1}[0, \infty]\right)\right]^{2}$. The latter enable us to conclude that either $\mu\left(z_{i}^{-1}[0, \infty]\right)=0$ or $\mu\left(z_{i}^{-1}[0, \infty]\right)=1$, i.e., either for $\mu$-a.e. $z_{i}(\omega)<0$ or $z_{i}(\omega) \geqq 0 \mu$-a.e.

Lemma 5.2. Let $\mathscr{E}$ be an exchange economy with differential information satisfying all the assumptions of Proposition 5.2. Then given a coalition $S$ where net trades $z_{i}(\cdot)$ are $\overline{\mathscr{F}}_{i}$-measurable for all $i \in S$ and $\sum_{i \in S} z_{i}(\omega)=0 \mu$-a.e., no trades can be beneficial to all $i$ in $S$ (i.e., $\int u_{i}\left(e_{i}+z_{i}\right) d \mu<\int u_{i}\left(e_{i}\right) d \mu$ for some $i$ in $S$ ).

Proof: By Lemma 5.2 for each $i \in S$, either $z_{i}(\omega) \geqq 0$ or $z_{i}(\omega)<0 \mu-$ a.e. We will show that whenever $z_{i}(\cdot)$ is either positive or negative for some $i$ in $S$ it will violate individual rationality. Since the case where $z_{i}(\omega)=0 \mu-a$.e. is trivial, we will only prove the case that for some $i \in S, z_{i}(\omega)>0 \mu-a . e$. (The case where for some $i \in S$, $z_{i}(\omega)<0 \mu-a . e$. can be proved along the same lines.) Since for some $i \in S$, $z_{i}(\omega)>0 \mu$-a.e. and $\sum_{i \in S} z_{i}(\omega)=0 \mu-a . e .$, it must be the case that for at least one $j \in S, z_{j}(\omega)<0 \mu-a . e$. Hence, $e_{j}(\omega)+z_{j}(\omega)<e_{j}(\omega) \mu-a . e$. By monotonicity we have that $u_{j}\left(e_{j}(\omega)+z_{j}(\omega)\right)<u_{j}\left(e_{j}(\omega)\right) \mu$-a.e. and therefore $\int u_{j}\left(e_{j}(\omega)+z_{j}(\omega)\right) d \mu(\omega)<$ $\int u_{j}\left(e_{j}(\omega)\right) d \mu(\omega)$. The above inequality violates individual rationality for the agent $j$ in $S$, and we can conclude that for coalition $S$, no net trade $z_{i}(\cdot)$ which is $\mathscr{F}_{i}$-measurable and $\sum_{i \in S} z_{i}(\omega)=0 \mu$-a.e. is beneficial, to all agents $i$ in $S$.

\section{The $\alpha$-core of an economy with differential information}

In this section we will allow for interdependent preferences (i.e., externalities in consumption). In particular, the economy $\mathscr{E}$ will be identical with that described in Sect. 3 except that now the utility function of each agent $i$, is a real valued function defined on $\prod_{j=1}^{n} Y_{j}$. Hence the utility function of each agent depends not 
only on his/her own consumption, but also on the consumption of all other agents. We will denote such an economy by $\Gamma$.

We now define the notion of a private $\alpha$-core for $\Gamma$ which corresponds to the private $\alpha$-core strategy for a normal form game which was defined in Yannelis (1991). We will first need some notation. If $S \subset I$ then $\left(y^{S}, z^{I \mid S}\right)$ denotes the vector $x$ in $\prod_{i \in I} Y_{j}$ where $x_{i}=y_{i}$ if $i \in S$ and $x_{i}=z_{i}$ if $i \notin S$.

Definition 6.1. The allocation $x: \Omega \rightarrow \prod_{i=1}^{n} X_{i}$ is said to be a private $\alpha$-core allocation for $\Gamma$ if the following conditions hold:

(i) Each $x_{i}: \Omega \rightarrow X_{i}$ is $\mathscr{F}_{i}$-measurable.

(ii) $\sum_{i=1}^{n} x_{i}(\omega)=\sum_{i=1}^{n} e_{i}(\omega) \mu$-a.e.

(iii) It is not true that there exist $S \subset I$ and $y: \Omega \rightarrow \prod_{i \in S} X_{i}$ such that each $y_{i}$ is $\mathscr{F}_{i}$-measurable for all $i \in S, \sum_{i \in S} y_{i}(\omega)=\sum_{i \in S} e_{i}(\omega) \mu-$ a.e. and $\int u_{i}\left(y^{S}, z^{I \mid S}\right) d \mu>\int u_{i}(x) d \mu$ for all $i \in S$ and for any $z^{I \mid S} \in \prod_{i \notin S} X_{i}$, each $z_{i}^{l \mid S}$ is $\mathscr{F}_{i}$-measurable for all $i \notin S$ and $\sum_{i \notin S} z_{i}(\omega)=\sum_{i \notin S} e_{i}(\omega) \mu$-a.e.

Conditions (i) and (ii) have been discussed in Section 3. (iii) says that no coalition can redistribute their initial resources (while each agent in the coalition is allowed to use his/her own private information) and make the expected utility of each member better off for any feasible redistribution of the complimentary coalition.

By replacing condition (iii) of Definition 6.1 by:

(iii') It is not true that there exist $S \subset I$ and $y: \Omega \rightarrow \prod_{i \in S_{i}}$ such that each $y_{i}$ is $\bigwedge_{i \in S} \mathscr{F}_{i}$-measurable for all $i \in S, \sum_{i \in S} y_{i}(\omega)=\sum_{i \in S} e_{i}(\omega) \mu-$ a.e. and $\int u_{i}\left(y^{S}, z^{I \mid S}\right) d \mu>$ $\int u_{i}(x) d \mu$ for all $i \in S$ and for any $z^{I \mid S} \in \prod_{i \neq S} X_{i}$, each $z_{i}^{I \mid S}$ is $\bigwedge_{i \notin S} \mathscr{F}_{i}$-measurable for all $i \notin S$ and $\sum_{i \notin S} z_{i}(\omega)=\sum_{i \notin S} e_{i}(\omega) \mu-$ a.e.,

we have the notion of a coarse $\alpha$-core allocation for $\Gamma$. Moreover if the measurability assumptions in (iii') above on $y_{i}$ and $z^{I I S}$ are replaced by: each $y_{i}$ is $\bigvee_{i \in S} \mathscr{F}_{i}$-measurable for all $i \in S$ and each $z_{i}^{l \mid S}$ is $\bigvee_{i \notin S} \mathscr{F}_{i}$-measurable for all $i \notin S$, we obtain the notion of a fine $\alpha$-core allocation for $\Gamma$.

In the next section we prove the existence of private $\alpha$-core allocations for $\Gamma$.

\section{The existence of private $\alpha$-core allocations}

We begin with some basic definitions of mathematical nature which will be needed for our existence proof.

\subsection{Mathematical preliminaries}

Let $(T, T, \mu)$ be a finite measure space and $X$ be a Banach space. Following Diestel-Uhl (1977) the function $f: T \rightarrow X$ is called simple if there exist $x_{1}, x_{2}, \ldots, x_{n}$ in $X$ and $\alpha_{1}, \alpha_{2}, \ldots, \alpha_{n}$ in $T$ such that $f=\sum_{i=1}^{n} x_{i} \chi_{\alpha_{i}}$, where $\chi_{\alpha_{i}}(t)=1$ if $t \in \alpha_{i}$ and 
$\chi_{\alpha_{i}}(t)=0$ if $t \notin \alpha_{i}$. A function $f: T \rightarrow X$ is said to be $\mu$-measurable if there exists a sequence of simple functions $f_{n}: T \rightarrow X$ such that $\lim _{n \rightarrow \infty}\left\|f_{n}(t)-f(t)\right\|=0$ for almost all $t \in T$. A $\mu$-measurable function $f: T \rightarrow X$ is said to be Bochner integrable if there exists a sequence of simple functions $\left\{f_{n}: n=1,2, \ldots\right\}$ such that

$$
\lim _{n \rightarrow \infty} \int_{T}\left\|f_{n}(t)-f(t)\right\| d \mu(t)=0 .
$$

In this case we define for each $E \in T$ the integral to be $\int_{E}(t) d \mu(t)=\lim _{n \rightarrow \infty} f_{n}(t) d \mu(t)$. It can be shown [see Diestel-Uhl (1977), Theorem 2, p. 45] that if $f: T \rightarrow X$ is a $\mu$-measurable function then, $f$ is Bochner integrable if and only if $\int_{T}\|f(t)\| d \mu(t)<\infty$. It is important to note that the Lebegue Dominated Convergence Theorem holds for Bochner integrable functions. In particular, if $f_{n}: T \rightarrow X,(n=1,2, \ldots)$ is a sequence of Bochner integrable functions such that $\lim _{n \rightarrow \infty} f_{n}(t)=f(t) \mu-a . e$., and $\left\|f_{n}(t)\right\| \leqq g(t) \mu-a . e$., (where $g: T \rightarrow \mathbb{R}$ is an integrable function), then $f$ is Bochner integrable and $\lim _{n \rightarrow \infty} \int_{T}\left\|f_{n}(t)-f(t)\right\| d \mu(t)=0$.

For $1 \leqq p<\infty$, we denote by $L_{p}(\mu, X)$ the space of equivalence classes of $X$-valued Bochner integrable functions $x: T \rightarrow X$ normed by

$$
\|x\|_{p}=\left(\int_{T}\|x(t)\|^{p} d \mu(t)\right)^{1 / p} .
$$

It is a standard result that normed by the functional \|\|$_{p}$ above, $L_{p}(\mu, X)$ becomes a Banach space [see Diestel-Uhl (1977), p. 50]. Recall that a correspondence $\phi$ : $T \rightarrow 2^{x}$ is said to be integrably bounded if there exists a map $h \in L_{1}(\mu, R)$ such that $\sup \{\|x\|: x \in \phi(t)\} \leqq h(t) \mu-$ a.e.

A Banach space $X$ has the Radon-Nikodym Property with respect to the measure space $(T, T, \mu)$ if for each $\mu$-continuous measure $G: T \rightarrow X$ of bounded variation there exists $g \varepsilon L_{i}(\mu, X)$ such that $G(E)=\int_{E} g(t) d \mu(t)$ for all $E \in T$. A Banach space $X$ has the Radon-Nikodym property (RNP) if $X$ has the RNP with respect to every finite measure space. Recall now [see Diestel-Uhl (1977, Theorem 1, p. 98)] that if $(T, T, \mu)$ is a finite measure space $1 \leqq p<\infty$, and $X$ is a Banach space, then $X^{*}$ has the RNP if and only if $\left(L_{p},(\mu, X)\right)^{*}=L_{q}\left(\mu, X^{*}\right)$ where $\frac{1}{p}+\frac{1}{q}=1$.

We now collect some basic results on Banach lattices [for an excellent treatment see Aliprantis-Burkinshaw (1985)]. A Banach lattice is a Banach space $L$ equipped with an order relation $\geqq$ (i.e., $\geqq$ is a reflexive, antisymmetric and transitive relation) satisfying:

(i) $x \geqq y$ implies $x+z \geqq y+z$ for every $z$ in $L$,

(ii) $x \geqq y$ implies $\lambda x \geqq \lambda y$ for all $\lambda \geqq 0$,

(iii) for all $x, y$ in $L$ there exists a supremum (least upper bound) $x \vee y$ and an infimum (greatest lower bound) $x \wedge y$,

(iv) $|x| \geqq|y|$ implies $\|x\| \geqq \mid y \|$ for all $x, y$ in $L$.

If $x, y$ are elements of the Banach lattice $L$, then we define the order interval $[x, y]$ 
as follows:

$$
[x, y]=\{z \in L: x \leqq z \leqq y\} .
$$

Note that $[x, y]$ is norm closed and convex (hence weakly closed). A Banach lattice $L$ is said to have an order continuous norm if, $x_{\alpha} \downarrow 0$ in $L$ implies $\left\|x_{\alpha}\right\| \downarrow 0$. A very useful result which will play an important role in the sequel is that if $L$ is a Banach lattice then the fact that $L$ has an order continuous norm is equivalent to weak compactness of the order interval $[x, z]=\{y \in L: x \leqq y \leqq z\}$ for every $x, z$ in $L$ [see Aliprantis-Burkinshaw (1985)].

We finally note that Cartwright (1974) has shown that if $X$ is a Banach lattice with an order continuous form (or equivalently $X$ has weakly compact order intervals) then $L_{1}(\mu, X)$ has weakly compact order intervals, as well. Cartwright's Theorem will play a crucial role in our existence proof.

\subsection{The private $\alpha$-core existence proof}

The following result provides sufficient conditions which guarantee the existence of a private $\alpha$-core allocation for $\Gamma$ where $|I|=2$. If $|I|>2$ then the result below is false.

Theorem 7.1. Let $\Gamma$ be an exchange economy with differential information satisfying for each $i,(i=1,2)$ the following assumptions.

(a.7.1) $X_{i}: \Omega \rightarrow 2^{Y}$ is a convex, closed, nonempty valued correspondence,

(a.7.2) $u_{i}: \prod_{j=1}^{n} Y_{j} \rightarrow \mathbb{R}$ is continuous, integrably bounded and concave.

Then a private $\alpha$-core allocation exists in $\Gamma$.

Proof. We first state the $\alpha$-core existence theorem in Yannelis (1991a) which will play a crucial role in our argument. Let $E=\left\{\left(X_{i}, u_{i}, e_{i}: i=1,2\right)\right\}$ be an exchange economy where

(1) $X_{i}$ the consumption set of agent $i$ is a subset of the positive cone of an ordered Hausdorff linear topological space $L$, which is endowed with a topology $\tau$ which is weaker than the Hausdorff topology on $L, \tau$ is a vector space topology having the property that all order intervals in $L$ are $\tau$-compact.

(2) the utility function of each agent $i, u_{i}: \prod_{j=1}^{n} X_{j} \rightarrow \mathbb{R}$ is concave and $\tau$-upper semi continuous.

(3) $e_{i}$ is the initial endowment of agent $i$, where $e_{i} \in X_{i}$ for all $i$.

If $E$ satisfies (1), (2), and (3) it follows from Yannelis (1991a, Theorem 4.1, p. 112) that an $\alpha$-core allocation exists ${ }^{12}$ in $E$.

\footnotetext{
${ }^{12}$ To be more specific, Yannelis (1991a) allows for preferences which need not be ordered. In particular one only needs to assume that the preference correspondence of agent $i P_{i}: \prod_{j=1}^{n} X_{j} \rightarrow 2 \prod_{i=1}^{n} X_{i}$ satisfies for each $i$ the following assumptions:

(i) $X_{i}=Y$,
} 
We now construct a new economy $\bar{\Gamma}$ as follows: For each $i$, $i=1,2$ let $L_{X_{i}}=\left\{x_{i} \in L_{1}(\mu, Y): x_{1}: \Omega \rightarrow Y\right.$ is Bochner integrable, $\mathscr{F}_{i}$-measurable and $x_{i}(\omega) \epsilon$ $X_{i}(\omega) \mu-$ a.e. $\}$. For each $i$ define $v_{i}: \prod_{j=1}^{n} L_{X_{j}} \rightarrow \mathbb{R}$ by $v_{i}(x)=\int u_{i}(x(\omega)) d \mu(\omega)$. Since by assumption $e_{i}$ is Bochner integrable $e_{i} \in L_{X_{i}}$ (recall that $e_{i}(\cdot)$ is $\mathscr{F}_{i}$-measurable and $e_{i}(\omega) \in X_{i}(\omega) \mu$-a.e.) and therefore $L_{X_{i}}$ is nonempty. Obviously $L_{X_{i}}$ it is convex and bounded from below. It follows from Balder-Yannelis (1991) that $v_{i}$ is weakly upper semicontinuous. Moreover, since $u_{i}$ is concave so is $v_{i}$. We now have a new economy $\bar{\Gamma}=\left\{\left(L_{X_{i}}, v_{i}, e_{i}\right): i=1,2\right\}$, where

(a) $L_{X_{i}}$ is the consumption set of agent $i$,

(b) $v_{i}$ is the utility function of agent $i$,

(c) $e_{i} \in L_{X_{i}}$ is the initial endowment of agent $i$.

It can be easily seen that an $\alpha$-core allocation of $\bar{\Gamma}$ is a private $\alpha$-core allocation for $\Gamma$. Hence all we need to show is that $\bar{\Gamma}$ satisfies the assumptions of Yannelis's (1991a) Theorem (i.e., conditions (1), (2), and (3) above).

Since $Y$ is the positive cone of a Banach Lattice with an order continuous norm, it follows from Cartwright's theorem that order intervals are weakly compact in $L_{1}(\mu, Y)$. Hence, the topology $\tau$ in Yannelis's (1991a) theorem is taken here to be the weak topology, and obviously assumption (1) is satisfied. Also as noted above $v_{i}$ is concave and weakly upper semicontinuous and $e_{i} \in L_{X_{i}}$ for all $i$. Hence (2) and (3) hold and therefore an $\alpha$-core allocation exists in $\bar{\Gamma}$. The latter implies that a private $\alpha$-core allocation exists in $\Gamma$. This completes the proof of the theorem.

Remark 7.1. One can easily see that the set of all private $\alpha$-core allocations for $\Gamma$ is contained in the set of all coarse $\alpha$-core allocations for $\Gamma$. However, for $|I|=2$ the coarse, fine and the private $\alpha$-core allocations coincide. From this observation and Theorem 7.1 we can obtain the following Corollary.

Corollary 7.1. Let $\Gamma$ be an exchange economy with differential information satisfying for each $i,(i=1,2)$ the assumptions of Theorem 7.1. Then a coarse and a fine $\alpha$-core allocation exists in $\Gamma$.

Remark 7.2. Recently Holly (1991) has shown that in an exchange economy

(ii) $x \notin \operatorname{con} P_{i}(x)$ for all $x \in \prod_{i=1}^{n} X_{i}$ (where con denotes convex hull),

(iii) $P_{i}$ has $\tau$-open lower sections (i.e., for every $y \in \prod_{i=1}^{n} X_{i}$, the set $P_{i}^{-1}(y)=\left\{x \in \prod_{i=1}^{n} X_{i}: y \in P_{i}(x)\right\}$ is $\tau$-open in $\left.\prod_{i=1}^{n} X_{i}\right)$.

Note now that by defining the preference correspondence $P_{i}: \prod_{j=1}^{n} X_{j} \rightarrow 2 \prod_{i=1}^{n} X_{i}$ by $P_{i}(x)=\left\{y: u_{i}(y)>u_{i}(x)\right\}$, it follows from the concavity of $u_{i}$ that $P_{i}(\cdot)$ is convex valued and clearly $x \notin \operatorname{con} P_{i}(x)=P(x)$ for all $x \in \prod_{i=1}^{n} X_{i}$. Moreover the $\tau$-upper semicontinuity of $u_{i}$-implies that $P_{i}$ has $\tau$-open lower sections. Hence, Theorem 4.1 of Yannelis (1991a) applies to the above setting. 
(without incomplete information) where the set of agents is greater than two, Yannelis's (1991a) $\alpha$-core existence theorem ceases to be true. It is straighforward to extend Holly's example to an exchange economy with differential information and show that if $|I|>2$ then the coarse $\alpha$-core of $\Gamma$ is empty, and therefore so is the private $\alpha$-core of $\Gamma$.

Remark 7.3. If the economy $\Gamma$ has one good per state, then the reader can easily verify that the private, coarse and fine $\alpha$-core allocations for $\Gamma$ are coalitional incentive compatible. The proof of this result is similar with that in Proposition 4.1.

\section{Conclusions}

The analysis of different core notions in an economy with differential information enables us to draw the following conclusions: The private core appears to be a sensible solution concept; it exists under very mild assumptions, it is coalitionally incentive compatible, and it takes into account the information superiority of an individual. Moreover, our examples indicate that it provides reasonable outcomes especially in situations where the traditional Walrasian equilibrium concept fails to do so. The coarse core appears to have the same properties as the private information core but since the latter concept is a strict subset of the former it does not provide any additional information. As our Example 5.1 indicated the coarse core is "too big." Contrary to the coarse core the fine core is "too small" and generally does not exist. However, whenever it exists (e.g., the weak fine core) it is not coalitionally incentive compatible and it does not take into account the information advantage of an agent. Nonetheless, we believe that the weak fine core may be useful for analyzing situations of adverse selection. We also showed (Sect. 7) that all the above core notions can be easily modified in order to allow for externalities in consumption. Since the private core can be used to explain intermediation, it is our belief that this concept has great potential in the theory of financial and incomplete markets. In particular, the fact that the private core rewards the agents with superior information provides interesting insights into the way that opportunities for financial intermediation or arbitrage arise in economies with differential information.

We conclude by noting that our adoption of a cooperative solution concept (e.g., the private core) to analyse economies with differential information seems to us very appealing. Indeed, in most applications agents cooperate either bilaterally or multilaterally under differential information. Although there is a non-cooperative feature in the private core notion, (i.e., private information sets are not verifiable by each member of a coalition), the resulting allocation is always coalitionally incentive compatible.

\section{A comparison with the value allocation}

Krasa-Yannelis (1991) examined the cardinal value allocation in an economy with differential information. Specifically, they analyzed the coarse, the fine, and the private value allocation. It was shown that the coarse and the fine value allocations 
are problematic (as is the case with the coarse and the fine core) but the private value allocation is coalitionally incentive compatible and it takes into account the information superiority of an individual. The latter two properties are shared by the private core as shown in this paper. Despite the fact that both concepts have the same appealing properties, (i.e., they are coalitionally incentive compatible and take into account the information superiority of an agent), they redistribute the initial endowments quite differently. In particular, a private value allocation need not be a private core allocation and vice versa. Thus, since the value and the core generate different outcomes, we cannot say whether one concept is better than the other. The decision for choosing the private value over the private core (or vice versa) should be based on the economic behavior that we intend to explain or rationalize. For instance, in modeling economic behavior where the bargaining power of an individual in a private information economy plays an important role the value seems in this situation more suitable that the core.

\section{References}

Aliprantis, C. D., Burkinshaw, O.: Positive operators. New York, London: Academic Press (1985)

Allen, B.: Market games with asymmetric information and nontransferable utility: representation results and the core. CARESS Working Paper 91-09, University of Pennsylvania (1991)

Balder, E. J., Yannelis, N. C.: On the weak continuity of expected utility. Mimeo (1991)

Berliant, M.: On income taxation and the core. J. Econ. Theory 56, 121-141 (1992)

Boyd, J., Prescott, E.C.: Financial intermediary coalitions. J. Econ. Theory 38, 211-232 (1986)

Cartwright, D.: The order completeness of some spaces of vector valued functions. Bull Aust. Math. Soc. 11, 57-61 (1974)

Diestel, J., Uhl, J. J.: Vector measures. In: Mathematical Surveys, vol. 15. Providence, Rhode Island:

American Mathematical Society (1977)

Holly, C.: Exchange economies can have an empty $\alpha$-core. Department of Mathematics, University of Illinois at Urbana-Champaign, Working Paper, (1991)

Kobayashi, T.: Equilibrium contracts for syndicates with differential information. Econometrica 48, 1635-1665 (1980)

Krasa, S., Yannelis, N. C.: The value allocation of an economy with differential information. University of Illinois at Urbana-Champaign, Working Paper, (1991)

Page, F. H.: Market games with differential information and infinite dimensional commodity spaces: the core. Mimeo, (1992)

Radner, R.: Equilibrium under uncertainty. Econometrica 36, 31-58 (1968)

Scafuri, A. J., Yannelis, N. C.: Non-symmetric cardinal value allocations. Econometrica 52, 1365-1368 (1984)

Wilson, R.: Information, efficiency, and the core of an economy. Econometrica 46, 807-816 (1978)

Yannelis, N. C.: The core of an economy with differential information. Econ. Theory 1, 183-198 (1991)

Yannelis, N. C.: The core of an economy without ordered preferences. In: Khan, M. A., Yannelis, N. C. (eds.) Equilibrium theory in infinite dimensioanl spaces. Springer Berlin, Heidelberg, New York: (1991a) 